\section{Cigarette smoking and thyroid eye disease: a systematic review}

J Thornton ${ }^{1}$, SP Kelly ${ }^{2}$, RA Harrison ${ }^{1,3}$ and R Edwards ${ }^{4}$ should be given effective support to stop smoking.

Eye (2007) 21, 1135-1145; doi:10.1038/sj.eye.6702603; published online 15 September 2006

Keywords: thyroid eye disease; Graves' ophthalmopathy; thyroid-associated ophthalmopathy; tobacco smoking; smoking cessation; epidemiology of the evidence using established causality criteria.

Results Fourteen papers describing 15 studies were included. There was a positive association between smoking and TED in four case-control studies when compared with control patients with Graves' disease but no ophthalmopathy (odds ratio (OR) 1.94-10.1) and in seven case-control studies in which control subjects did not have thyroid disease (OR 1.22-20.2). Two cohort studies examined the occurrence of new cases of TED; one study found an increased incidence of TED with smoking. Four cohort studies investigated progression or outcome of treatment in patients with established TED, three finding an association between smoking and poorer outcome. The quality of the studies was variable, but the association with smoking was strong in the most methodologically rigorous studies. Other evidence supporting a causal link was a consistent association across studies, a dose-response effect, a reduced risk of TED in ex-smokers, and a temporal relationship.

Conclusion This systematic review provided strong evidence for a causal association between smoking and development of TED. Current-smokers were also more likely to experience disease progression or poorer outcome of treatment. Patients with Graves' disease and the general public should be educated about the risk of smoking and TED. These findings suggest that patients with Graves' disease or TED who are smokers

\section{Introduction}

Thyroid eye disease (TED), also known as Graves' ophthalmopathy or thyroid-associated ophthalmopathy, is the most frequent extrathyroidal manifestation of autoimmune hyperthyroidism (Graves' disease); 25-50\% of patients with Graves' disease have eye involvement, of whom 3-5\% develop severe disease. ${ }^{1,2}$ The aetiology of TED is complex. Genetic susceptibility and endogenous and environmental factors play a role in its development. $^{2-4}$ The genetic factors are poorly defined but appear to have the lesser role. ${ }^{2}$ The risk of developing TED increases with age and women are more likely to develop Graves' disease than men. ${ }^{4}$

An association of tobacco smoking with TED was first described in $1987^{5}$ and the evidence to support a causal link is growing. ${ }^{6}$ Three recent reviews concluded there is a causal association. $^{7-9}$ However, none of these previous reviews included non-English language papers or an appraisal of the quality of the included studies and two did not state the methods for the literature search and identification of studies., ${ }^{7,8}$ Therefore, we aimed to expand on this previous work and undertake a systematic review of the evidence, including a rigorous quality assessment of studies and application of standard criteria for causality, to robustly assess the relationship between smoking and TED.
${ }^{1}$ Evidence for Population Health Unit, University of Manchester Manchester, UK

${ }^{2}$ Ophthalmology Department, Bolton Hospitals NHS Trust, Bolton, UK

${ }^{3}$ Bolton Primary Care Trust, Bolton, UK

${ }^{4}$ Department of Public Health, Wellington School of Medicine and Health Sciences, Wellington, New Zealand

Correspondence:

J Thornton,

Evidence for Population

Health Unit,

University of Manchester, Oxford Road,

Manchester M13 9PT, UK Tel: + 4401612757146 Fax: + 4401612755043 E-mail: judith.thornton@ manchester.ac.uk

Received: 25 June 2006 Accepted in revised form: 9 August 2006 Published online: 15 September 2006

The authors have no competing interests to declare 


\section{Materials and methods}

\section{Identification of studies}

MEDLINE (on Ovid from 1966) and EMBASE (on Ovid from 1980) were searched as well as ISI Web of Science (from 1990). Keywords and MESH terms for 'randomised controlled trials' or 'observational studies' were combined with 'tobacco smoking' and 'thyroid eye disease' or its similes. The final list of titles and abstracts was screened by two reviewers (JT and RE) and full publications obtained where articles were thought to be potentially relevant. Bibliographies of included studies and review papers were screened to identify other relevant studies. The main searches were run in September 2003 and March 2005 and updated in October 2005.

\section{Inclusion and exclusion criteria}

Analytical observational epidemiological studies (case-control and cohort studies) and intervention studies with comparison groups were included if they had measurements of tobacco-smoking status, and reported the association between smoking and the development, progression, or treatment outcomes of TED after appropriate statistical analysis. We excluded crosssectional studies as they are less appropriate for assessing evidence of association where the temporal relationship between exposure and outcome and direction of causality is uncertain. ${ }^{10}$ We excluded conference abstracts as they contain insufficient detail. Non-English language papers were translated as necessary.

\section{Quality assessment and data extraction}

We did not use an established quality assessment tool. A previous review of quality assessment tools for nonrandomised studies found that none addressed all the key dimensions of quality. ${ }^{11}$ We investigated a range of possible tools for observational epidemiological studies, but found none which addressed all the key areas of the design and conduct of studies, nor included detailed and specific enough criteria and scoring systems to assess the quality of studies investigating the link between smoking and TED. For example, we considered that precise detailed criteria were essential for evaluating key quality dimensions such as the adequacy of the process of identifying and defining cases or progression of disease, and of smoking exposure status assessment. We therefore developed separate case-control and cohort study appraisal tools adapted initially from existing instruments. ${ }^{12-15}$ We further refined these tools through a process of discussion and review within our team, which was carrying out a series of systematic reviews of the link between smoking and eye disease. This team had relevant epidemiology and ophthalmology expertise.

The resulting review tools (available on request) aimed to assess all the key dimensions of quality graded generally as 'fully', 'partially', or 'not' met. The tools included detailed descriptions of the methodological quality criteria required for a study to 'fully' or 'partially' meet each quality criteria. There were 12 quality criteria for case-control studies and 14 for cohort studies (Table 1). Five of these criteria (marked in bold in Table 1) were classed as 'key criteria' based on discussions within the team and review of standard epidemiological texts to identify critical potential areas of weakness of each design.

Two authors (JT and RE) independently reviewed the identified papers, extracted the data, and completed the quality assessment. Results were compared and discrepancies discussed with adjudication by the third reviewer (SPK) if required. We set $a$ priori eligibility criteria to determine if studies were eligible for inclusion in a meta-analysis. Studies that included suitable data and at least partially met all five key quality criteria were eligible. Meta-analysis was not possible because only one study $^{16}$ was of adequate quality. Findings were summarised using tables and narrative synthesis.

\section{Results}

\section{Literature search}

We identified 181 papers from the literature databases and five additional papers from screening bibliographies. After initial screening, 44 potential studies were identified, but 26 of these studies did not meet the inclusion criteria. We excluded a further four studies during the quality review process because measures of association between smoking and TED were not presented and there was insufficient data provided for them to be calculated by the reviewers. ${ }^{17-20}$ One of the included papers described two separate cohort studies. ${ }^{21}$ Therefore, the review included 14 papers describing 15 studies (nine case-control and six cohort).

\section{Description of included studies}

Eleven studies were conducted in Europe, ${ }^{5,16,21-28}$ two in the US, ${ }^{29,30}$ and one each in Taiwan ${ }^{31}$ and Japan. ${ }^{32}$ Most included more female than male subjects. Ages of subjects ranged from around 20 years to around 80 years. Seven papers did not present odds ratios (ORs) and unadjusted ORs were calculated by reviewers (RE/RH) from data in the papers. ${ }^{21,22,24-27,29}$ In addition, the $95 \%$ confidence intervals (CIs) were calculated for one study. ${ }^{32}$ 
Table 1 Quality criteria for assessment of case-control and cohort studies included in the review: key quality criteria are marked in bold

\begin{tabular}{|c|c|}
\hline Case-control studies & Cohort studies \\
\hline Degree to which study base and time period described & Degree to which study base and time period described \\
\hline Representativeness of controls of the study base & Representativeness and comparability of the cohort(s) \\
\hline $\begin{array}{l}\text { Adequacy of process for identification and definition of } \\
\text { cases or progression/response to treatment }\end{array}$ & $\begin{array}{l}\text { Adequacy of process for identification and definition of cases } \\
\text { or progression/response to treatment }\end{array}$ \\
\hline Representativeness of cases eligible for inclusion & Equivalence of case ascertainment procedures for exposure groups \\
\hline Adequacy of response rates & Adequacy of response rates \\
\hline Adequacy of data collection methods & Adequacy of data collection methods \\
\hline Adequacy of data on smoking history & Adequacy of data on smoking history \\
\hline $\begin{array}{l}\text { Equivalence of data collection methods in cases } \\
\text { and controls }\end{array}$ & $\begin{array}{l}\text { Adequacy of allocation into exposure groups by smoking status } \\
\text { Equality of follow-up between exposure groups }\end{array}$ \\
\hline Adequacy of main analysis methods & Adequacy of length of follow-up period \\
\hline Adequacy of exposure variables used in analysis. & Adequacy of main analysis methods \\
\hline $\begin{array}{l}\text { Adequacy of dealing with potential confounders } \\
\text { through design or analysis }\end{array}$ & $\begin{array}{l}\text { Adequacy of exposure variables used in analysis. } \\
\text { Adequacy of dealing with potential confounders through design } \\
\text { or analysis }\end{array}$ \\
\hline
\end{tabular}

Two studies presented $P$-values from another acceptable method of analysis of association, Kaplan-Meier life-table analysis. ${ }^{28,30}$

Eight of the case-control studies (Table 2) recruited patients with prevalent TED. One case-control study recruited incident cases. ${ }^{24}$ Incident cases are preferred because with prevalent cases smoking status or recall of smoking status may change after development of TED.

In the case-control studies, two main comparisons were used: comparing smoking status or history between TED cases and non-Graves' disease controls (eight studies), and between TED cases and patients with Graves' disease but no TED (five studies). Several studies included both types of comparison by using more than one control group. The comparison of smoking in TED cases and patients with Graves' disease but without TED is much more informative as it provides specific evidence of whether smoking is associated with the development of TED in patients with Graves' disease. Comparisons of smoking between TED cases and non-Graves' disease controls are more difficult to interpret as a positive association could imply a relationship of smoking with the development of Graves' disease or TED or both. The studies which recruited controls without Graves' disease did so from either the community ${ }^{24,29,31}$ or from hospital clinics $^{22,23,25,32}$ or from both. ${ }^{5}$ The studies which used

Graves' disease controls recruited controls from hospital clinics. ${ }^{5,22,24-26}$

Four prospective and two retrospective cohort studies investigated the incidence, progression, or treatment outcomes by smoking status among cohorts of patients with Graves' disease (Table 3). One study assessed the incidence of TED in patients with Graves' disease of recent onset. ${ }^{16}$ Another reported both the incidence of
TED and progression of existing TED. ${ }^{27}$ Four studies examined progression and response to treatment of TED by smoking status. ${ }^{21,27,28,30}$

\section{Quality assessment}

The quality of the included studies varied and was often poor. None of the case-control studies met all five key quality criteria. Of the five case-control studies which used controls with Graves' disease without TED, one fully or partially met all of the key quality criteria except, the method of analysis; ${ }^{22}$ three studies fully or partially met three criteria ${ }^{24-26}$ and one met only two criteria. ${ }^{5}$ Of the other four case-control studies which only used controls without Graves' disease, one fully or partially met four key criteria and also at least partially met 11 out of 12 of all the quality criteria. ${ }^{23}$

Across all nine case-control studies, the smoking variable used in the analysis was adequate in only one study. ${ }^{22}$ The main problem was that smoking status was very crudely and inadequately categorised with ex-smokers combined with current-smokers, or split between current- and non-smokers according to when quitting occurred. If there is an association of TED with smoking that is attenuated by quitting smoking, these approaches will tend to reduce the size of the observed association between TED and smoking.

Only two studies fully met the criterion for the quality of the analysis. ${ }^{5,31}$ All but one of the studies at least partially met the criterion for case identification and definition, although none fully met this criterion because all but one study used prevalent rather than incident cases and only four studies reported use of a standard classification system for TED. ${ }^{5,23,31,32}$ All seven of the 
Table 2 Thyroid eye disease and smoking: summary of case-control studies

\begin{tabular}{|c|c|c|c|c|c|c|c|}
\hline Study & $\begin{array}{l}\text { Study population, } \\
\text { location }\end{array}$ & Case characteristics & $\begin{array}{l}\text { Control } \\
\text { characteristics }\end{array}$ & $\begin{array}{l}\text { Exposure } \\
\text { measurement, } \\
\text { assessment } \\
\text { smoking status }\end{array}$ & Analysis variables & $\begin{array}{l}\text { Confounders } \\
\text { addressed } \\
\text { through design } \\
\text { or analysis }\end{array}$ & $\begin{array}{l}\text { Association of smoking with } \\
\text { thyroid eye disease }\end{array}$ \\
\hline $\begin{array}{l}\text { Hagg and } \\
\text { Asplund }^{5}\end{array}$ & $\begin{array}{l}\text { Cases: University } \\
\text { Hospital, Sweden } \\
\text { Controls: } \\
\text { 1. Community } \\
\text { (population } \\
\text { register), Sweden } \\
\text { 2. Hospital clinic, } \\
\text { Sweden }\end{array}$ & $\begin{array}{l}\text { Prevalent } \\
12 \text { endocrine } \\
\text { ophthalmopathy } \\
\text { ( } \geq 3 \text { Werner's } \\
\text { classification), } 2 \mathrm{M} \\
10 \mathrm{~F} \text {, mean age onset } \\
\text { ophthalmopathy } 38 \\
\text { (range 24-51) years }\end{array}$ & $\begin{array}{l}\text { 1. Forty-two } \\
\text { community controls } \\
\text { (no thyroid } \\
\text { disorder) } \\
\text { 2. Twenty-four } \\
\text { subjects with GD } \\
\text { but slight or no eye } \\
\text { disease, matched } \\
\text { for age and sex }\end{array}$ & $\begin{array}{l}\text { Questionnaire } \\
\text { Current smoking } \\
\text { status and at onset } \\
\text { of disease }\end{array}$ & $\begin{array}{l}\text { Current-smokers } \\
\text { (those who had } \\
\text { smoked for some } \\
\text { period during the } \\
\text { past year) vs } \\
\text { non-smokers. } \\
\text { Current-smokers } \\
\text { plus ex-smokers } \\
\text { (those who had } \\
\text { been smokers but } \\
\text { had not smoked in } \\
\text { the past year) vs } \\
\text { never-smokers }\end{array}$ & Sex, age & $\begin{array}{l}\text { Current-smokers vs non- } \\
\text { smokers, TED cases and GD } \\
\text { controls: adjusted OR } 10.0 \\
\text { (95\% CI 1.4-74.3) } \\
\text { Current-smokers plus ex- } \\
\text { smokers vs never-smokers, } \\
\text { TED cases and GD controls: } \\
\text { adjusted OR } 4.5 \text { (95\% } \\
\text { CI 0.8-24.5) } \\
\text { Current-smokers vs non- } \\
\text { smokers, TED cases and } \\
\text { community controls: adjusted } \\
\text { OR } 20.2 \text { (95\% CI 2.8-144.8) } \\
\text { Current-smokers plus ex- } \\
\text { smokers vs never-smokers, } \\
\text { TED cases and community } \\
\text { controls: adjusted OR } 8.9 \\
\text { (95\% CI 1.3-60.2) }\end{array}$ \\
\hline Shine et $a l^{22}$ & $\begin{array}{l}\text { Cases: Specialist } \\
\text { Eye Hospital } \\
\text { (London, UK) } \\
\text { Controls: Specialist } \\
\text { Eye Hospital } \\
\text { (London, UK) and } \\
\text { Teaching Hospital } \\
\text { (Cambridge, UK) }\end{array}$ & $\begin{array}{l}\text { Prevalent } \\
85 \text { severe TED (van } \\
\text { Dyck classification), } \\
\text { median age } 68 \\
\text { (range 21-80) years }\end{array}$ & $\begin{array}{l}\text { 1. Sixty-two subjects } \\
\text { with GD median } \\
\text { age } 56 \text { (range 15-81) } \\
\text { years } \\
\text { 2. Eighty-one } \\
\text { subjects with other } \\
\text { thyroid disease or } \\
\text { no thyroid disease, } \\
\text { median age } 61 \\
\text { (25-91) years }\end{array}$ & $\begin{array}{l}\text { Questionnaire } \\
\text { Lifelong smoking } \\
\text { status }\end{array}$ & $\begin{array}{l}\text { Current-smokers } v s \\
\text { never-smokers } \\
\text { (no definitions) }\end{array}$ & None & $\begin{array}{l}\text { TED cases and GD controls: } \\
\text { OR } 3.78 \text { ( } 95 \% \text { CI 1.63-9.05) } \\
\text { (calc by reviewer) } \\
\text { TED cases and GD plus } \\
\text { other controls: OR } 9.42 \text { ( } 95 \% \\
\text { CI } 3.85-23.7 \text { (calc by reviewer) }\end{array}$ \\
\hline $\begin{array}{l}\text { Prummel } \\
\text { and } \\
\text { Wiersinga }^{23}\end{array}$ & $\begin{array}{l}\text { Cases: } \\
\text { Endocrinology } \\
\text { Clinic, the } \\
\text { Netherlands } \\
\text { Controls: General } \\
\text { Medicine Clinics, } \\
\text { the Netherlands }\end{array}$ & $\begin{array}{l}\text { Prevalent } \\
100 \text { TED with onset } \\
\text { of disease within } 10 \\
\text { years of study and } \\
\text { no thyrotoxicosis, } \\
16 \mathrm{M} 84 \mathrm{~F} \text {, mean age } \\
46.4 \pm 12.8 \text { years }\end{array}$ & $\begin{array}{l}\text { Four hundred } \\
\text { hospital and } \\
\text { community controls } \\
\text { 1. Patients visiting } \\
\text { general ophthalmic } \\
\text { outpatient clinic. } \\
\text { 2. Individuals } \\
\text { accompanying } \\
\text { patients to } \\
\text { endocrine clinic }\end{array}$ & $\begin{array}{l}\text { Self-completion } \\
\text { questionnaire } \\
\text { Smoking status } \\
\text { assessed at onset } \\
\text { of disease }\end{array}$ & $\begin{array}{l}\text { Smokers (current- } \\
\text { smokers and those } \\
\text { who had stopped } \\
\text { within } 5 \text { years) vs } \\
\text { non-smokers (non- } \\
\text { smokers or ceased } \\
\text { more than } 5 \text { years } \\
\text { previously) }\end{array}$ & Sex, age & $\begin{array}{l}\text { Adjusted OR } 6.5 \\
\text { (95\% CI 3.8-11.2) }\end{array}$ \\
\hline
\end{tabular}


Table 2 (Continued)

\begin{tabular}{|c|c|c|c|c|c|c|c|}
\hline Study & $\begin{array}{l}\text { Study population, } \\
\text { location }\end{array}$ & Case characteristics & $\begin{array}{l}\text { Control } \\
\text { characteristics }\end{array}$ & $\begin{array}{l}\text { Exposure } \\
\text { measurement, } \\
\text { assessment } \\
\text { smoking status }\end{array}$ & Analysis variables & $\begin{array}{l}\text { Confounders } \\
\text { addressed } \\
\text { through design } \\
\text { or analysis }\end{array}$ & $\begin{array}{l}\text { Association of smoking with } \\
\text { thyroid eye disease }\end{array}$ \\
\hline Winsa et $a l^{24}$ & $\begin{array}{l}\text { Cases: County } \\
\text { hospitals, Sweden } \\
\text { Controls: } \\
\text { Community, } \\
\text { Sweden }\end{array}$ & $\begin{array}{l}\text { Incident } \\
62 \text { TED (grades I- } \\
\text { IV), } 13 \mathrm{M} 49 \mathrm{~F} \text {, mean } \\
\text { age } 44 \pm 12 \text { years }\end{array}$ & $\begin{array}{l}\text { Three hundred and } \\
\text { seventy-two healthy } \\
\text { community } \\
\text { controls; } 142 \text { GD } \\
\text { and no } \\
\text { ophthalmopathy }\end{array}$ & $\begin{array}{l}\text { Self-completion } \\
\text { questionnaire } \\
\text { Time of smoking } \\
\text { status assessment } \\
\text { not stated }\end{array}$ & $\begin{array}{l}\text { Ever-smokers } \\
\text { (current and } \\
\text { former smokers) } \\
v s \text { non-smokers }\end{array}$ & Sex, age & $\begin{array}{l}\text { Current-smokers } v s \text { non- } \\
\text { smokers TED cases and GD no } \\
\text { TED controls: OR } 1.53 \text { ( } 95 \% \text { CI } \\
\text { 0.83-2.80) (calc by reviewer) } \\
\text { Ever-smokers } v \text { s never- } \\
\text { smokers, TED cases and GD } \\
\text { no TED controls: OR } 2.07 \\
\text { (95\% CI 1.12-3.84) (calc by } \\
\text { reviewer) } \\
\text { Current-smokers vs non- } \\
\text { smokers, TED cases and } \\
\text { community controls: OR } 2.20 \\
\text { (95\% CI 1.28-3.80 (calc by } \\
\text { reviewer) } \\
\text { Ever-smokers vs never- } \\
\text { smokers, TED cases and } \\
\text { community controls: OR } 2.35 \\
\text { (95\% CI 1.35-4.09) (calc by } \\
\text { reviewer) }\end{array}$ \\
\hline $\begin{array}{l}\text { Nunery } \\
\text { et } a l^{29}\end{array}$ & $\begin{array}{l}\text { Cases: University } \\
\text { Eye Institute, US } \\
\text { Controls: } \\
\text { Community, US }\end{array}$ & $\begin{array}{l}\text { Prevalent } \\
\text { TED categorised as } \\
\text { type I (no restrictive } \\
\text { diplopia) or type II } \\
\text { (restrictive diplopia } \\
\text { present). } \\
\text { Type I: } 56 \mathrm{~F} 9 \mathrm{M} \text {, } \\
\text { mean age } 36 \pm 12.5 \\
\text { years } \\
\text { Type II: } 38 \mathrm{~F} 21 \mathrm{M} \text {, } \\
\text { mean age } 52 \pm 11.7 \\
\text { years }\end{array}$ & $\begin{array}{l}\text { Community } \\
\text { controls identified } \\
\text { through random } \\
\text { telephone number } \\
\text { dialling. Age range } \\
\text { 25-64 years }\end{array}$ & $\begin{array}{l}\text { Questionnaire for } \\
\text { cases, telephone } \\
\text { interview for } \\
\text { controls } \\
\text { Smoking status } \\
\text { assessed at onset for } \\
\text { cases, current status } \\
\text { for control }\end{array}$ & $\begin{array}{l}\text { Current-smokers } \\
\text { (within } 2 \text { years } \\
\text { of onset) vs } \\
\text { non-smokers }\end{array}$ & Sex, age & $\begin{array}{l}\text { Type I TED cases and } \\
\text { community controls: OR } 3.91 \\
\text { (calc by reviewer) } \\
\text { Type II TED cases and } \\
\text { community controls: OR } 13.5 \\
\text { (calc by reviewer) }\end{array}$ \\
\hline $\begin{array}{l}\text { O'Hare and } \\
\text { Geoghegan }\end{array}$ & $\begin{array}{l}\text { Cases: } \\
\text { Endocrinology } \\
\text { Clinic, Ireland } \\
\text { Controls: } \\
\text { Orthopaedic and } \\
\text { Trauma Clinic, } \\
\text { Ireland }\end{array}$ & $\begin{array}{l}\text { Prevalent } \\
34 \text { TED cases : } 5 \mathrm{M} \\
29 \mathrm{~F} \text {, mean age } \\
37 \pm 16 \text { years }\end{array}$ & $\begin{array}{l}\text { Hospital: } 168,26 \mathrm{M} \\
142 \mathrm{~F} \text { with other } \\
\text { thyroid diseases } \\
\text { mean age } 43 \pm 17 \\
\text { years } \\
\text { GD no TED } \\
\text { controls: } 34,8 \mathrm{M} \\
41 \mathrm{~F} \text {, mean age } \\
44 \pm 16 \text { years }\end{array}$ & $\begin{array}{l}\text { Structured } \\
\text { interview } \\
\text { Lifelong assessment } \\
\text { of smoking status }\end{array}$ & $\begin{array}{l}\text { Ever-smokers vs } \\
\text { never-smokers, } \\
\text { smoker before } \\
\text { onset disease vs } \\
\text { never-smokers }\end{array}$ & $\begin{array}{l}\text { Sex, age, } \\
\text { thyrotoxicosis }\end{array}$ & $\begin{array}{l}\text { TED cases and GD controls. } \\
\text { Smoking at onset } v s \text { non- } \\
\text { smokers: OR } 1.40 \text { ( } 95 \% \\
\text { CI 0.5-4.03) (calc by reviewer) } \\
\text { TED cases and non GD } \\
\text { controls. Ever-smokers } v s \\
\text { never-smokers: OR } 2.29 \\
\text { (95\% CI 1.03-5.08) (calc by } \\
\text { reviewer) }\end{array}$ \\
\hline
\end{tabular}


Table 2 (Continued)

\begin{tabular}{|c|c|c|c|c|c|c|c|}
\hline Study & $\begin{array}{l}\text { Study population, } \\
\text { location }\end{array}$ & Case characteristics & $\begin{array}{l}\text { Control } \\
\text { characteristics }\end{array}$ & $\begin{array}{l}\text { Exposure } \\
\text { measurement, } \\
\text { assessment } \\
\text { smoking status }\end{array}$ & Analysis variables & $\begin{array}{l}\text { Confounders } \\
\text { addressed } \\
\text { through design } \\
\text { or analysis }\end{array}$ & $\begin{array}{l}\text { Association of smoking with } \\
\text { thyroid eye disease }\end{array}$ \\
\hline & & & & & & & $\begin{array}{l}\text { TED cases and non-GD } \\
\text { controls. Smoking at onset } v s \\
\text { never-smokers: OR } 3.48 \\
\text { ( } 95 \% \text { CI 1.61-7.52) (calc by } \\
\text { reviewer) }\end{array}$ \\
\hline $\begin{array}{l}\text { Bartalena } \\
\text { et } a l^{26}\end{array}$ & $\begin{array}{l}\text { Cases: } \\
\text { Endocrinology } \\
\text { Clinic, University } \\
\text { Hospitals, Italy } \\
\text { Controls: } \\
\text { Endocrinology } \\
\text { Clinics, Italy }\end{array}$ & $\begin{array}{l}\text { Prevalent } \\
307 \text { TED cases, all F, } \\
\text { mean age } 46.2 \\
\text { (range } 16-74 \text { ) years }\end{array}$ & $\begin{array}{l}\text { Hospital } \\
167 \text { GD without } \\
\text { TED. All F. Mean } \\
\text { age } 45.4 \text { (range } \\
\text { 17-73) years, } 486 \mathrm{~F} \\
\text { endocrine clinic } \\
\text { attenders with no } \\
\text { thyroid abnormality } \\
\text { mean age } 37.3 \\
\text { (range 15-77) years }\end{array}$ & $\begin{array}{l}\text { Questionnaire } \\
\text { Lifelong assessment } \\
\text { of smoking status }\end{array}$ & $\begin{array}{l}\text { Ever-smokers vs } \\
\text { never-smokers }\end{array}$ & Sex age & $\begin{array}{l}\text { TED cases and GD controls, } \\
\text { OR } 1.94 \text { ( } 95 \% \text { CI 1.3-2.91) } \\
\text { (calc by reviewer) } \\
\text { TED cases and non GD } \\
\text { hospital controls: OR } 4.65 \\
\text { (95\% CI 3.39-6.4) (calc by } \\
\text { reviewer) } \\
\text { TED cases and all controls: } \\
\text { OR } 4.22 \text { ( } 95 \% \text { CI 3.17-5.66) } \\
\text { (calc by reviewer) }\end{array}$ \\
\hline $\begin{array}{l}\text { Shiraishi } \\
\text { et } a l^{32}\end{array}$ & $\begin{array}{l}\text { Cases: Hospital } \\
\text { Clinics, Japan } \\
\text { Controls: Internal } \\
\text { Medicine } \\
\text { Department, Japan }\end{array}$ & $\begin{array}{l}\text { Prevalent } \\
102 \text { TED cases, } 18 \mathrm{M} \\
84 \mathrm{~F} \text {, mean age } \\
\mathrm{M} 46.1 \text { (range } \\
\text { 17-77) years, mean } \\
\text { age F } 39.4 \text { (range } \\
\text { 17-77) years }\end{array}$ & $\begin{array}{l}\text { Hospital controls } \\
\text { (unspecified). 102, } \\
18 \text { M } 84 \text { F. Mean age } \\
\text { M } 46.1 \text { (range } \\
\text { 17-77) years, mean } \\
\text { age F } 39.5 \text { (range } \\
\text { 17-77) years }\end{array}$ & $\begin{array}{l}\text { Clinical record, } \\
\text { or if absent } \\
\text { questionnaire } \\
\text { Lifelong assessment } \\
\text { of smoking status }\end{array}$ & $\begin{array}{l}\text { Smoker/recent ex- } \\
\text { smoker (<5 years } \\
\text { before onset) } v s \\
\text { never-smoker/long- } \\
\text { term ex-smoker ( }>5 \\
\text { years before onset) }\end{array}$ & Sex, age & $\begin{array}{l}\text { OR } 1.22 \text { (95\% CI } 0.66-2.26) \\
\text { (95\% CI calc by reviewer) }\end{array}$ \\
\hline
\end{tabular}

$\mathrm{GO}=$ Graves' ophthalmopathy, $\mathrm{GD}=$ Graves' disease, $\mathrm{TED}=$ thyroid eye disease. 
Table 3 Thyroid eye disease and smoking: summary of cohort studies

\begin{tabular}{|c|c|c|c|c|c|c|c|c|}
\hline Study & $\begin{array}{l}\text { Study population, } \\
\text { location }\end{array}$ & $\begin{array}{l}\text { Study type and } \\
\text { follow-up period }\end{array}$ & Cohort characteristics & Outcomes assessed & Exposure measurement & Analysis variables & $\begin{array}{l}\text { Confounders addressed } \\
\text { through design or } \\
\text { analysis }\end{array}$ & $\begin{array}{l}\text { Association of smoking with } \\
\text { thyroid eye disease }\end{array}$ \\
\hline Tallstedt $e t a l^{27}$ & $\begin{array}{l}\text { Hospital Clinics, } \\
\text { Sweden }\end{array}$ & $\begin{array}{l}\text { Prospective study of } \\
\text { patients within GD } \\
\text { clinical trial, } \\
>2 \text { years follow-up }\end{array}$ & $\begin{array}{l}\text { One hundred and } \\
\text { seventy-one GD patients } \\
\text { with and without TED from } \\
\text { clinical trial } \\
\text { Smokers: } 89 \text { total, } 70 \text { without } \\
\text { TED at baseline. } 18 \mathrm{M} 71 \mathrm{~F} \text {, } \\
\text { age range } 25-55 \text { years } \\
\text { Non-smokers: } 82 \text { total, } 77 \\
\text { without TED at baseline. } 11 \mathrm{M} \\
71 \mathrm{~F} \text {, age range } 25-55 \text { years }\end{array}$ & $\begin{array}{l}\text { Incidence of new } \\
\text { cases of TED and } \\
\text { progression existing } \\
\text { disease }\end{array}$ & $\begin{array}{l}\text { Self-completion } \\
\text { questionnaire } \\
\text { Lifelong assessment of } \\
\text { smoking status }\end{array}$ & $\begin{array}{l}\text { Current-smokers } \\
\text { (including ex-smokers } \\
<1 \text { year) vs non-smokers } \\
\text { (including ex-smokers } \\
>1 \text { year) }\end{array}$ & Thyroid dysfunction & $\begin{array}{l}\text { RR } 1.59 \text { (95\% CI } 0.72-3.49) \\
\text { (calc by reviewer) }\end{array}$ \\
\hline $\begin{array}{l}\text { Pfeilschifter and } \\
\text { Ziegler }^{16}\end{array}$ & $\begin{array}{l}\text { University } \\
\text { Department of } \\
\text { Endocrinology and } \\
\text { Metabolism, Germany }\end{array}$ & $\begin{array}{l}\text { Prospective } \geq 1 \text { year } \\
\text { follow-up }\end{array}$ & $\begin{array}{l}\text { Two hundred and fifty-three } \\
\text { patients with GD of recent } \\
\text { onset. } 212 \mathrm{~F} 41 \mathrm{M} \text {, mean age } \\
36.8 \pm 12.4 \text { years. } \\
\text { Smokers: } 87 \mathrm{~F} 17 \text {, mean age } \\
35.9 \pm 10.8 \text { years. } \\
\text { Never-smokers: } 107 \mathrm{~F} 17 \\
\text { mean age } 36.8 \pm 13.3 \text { years } \\
\text { Ex-smokers: } 18 \mathrm{~F} 7 \mathrm{M} \\
40.0 \pm 13.7 \text { years. }\end{array}$ & $\begin{array}{l}\text { Incidence of new } \\
\text { cases of TED: total } \\
\text { ophthalmopathy, } \\
\text { proptosis, diplopia }\end{array}$ & $\begin{array}{l}\text { Questionnaire } \\
\text { Lifelong assessment of } \\
\text { smoking status }\end{array}$ & $\begin{array}{l}\text { Current-smokers } v s \\
\text { never-smokers, } \\
\text { ex-smokers }>20 \\
\text { cigs/day vs-never } \\
\text { smoker }\end{array}$ & $\begin{array}{l}\text { Sex, age ethnicity, } \\
\text { surgery/radiotherapy }\end{array}$ & $\begin{array}{l}\text { Total ophthalmopathy: Current- } \\
\text { smokers vs never-smokers: RR } 1.32 \\
\text { (95\% CI 1.06-1.64) } P=0.011 \\
\text { Proptosis: current-smokers vs } \\
\text { never-smokers: RR 2.64 (95\% CI } \\
\text { 1.79-3.90) } P<0.0001 \\
\text { Diplopia: Current-smokers vs } \\
\text { never-smokers: RR } 3.14 \text { (95\% CI } \\
\text { 1.65-5.98) } P<0.0001\end{array}$ \\
\hline Bartley et $a l^{30}$ & $\begin{array}{l}\text { Population-based, } \\
\text { cohort from } \\
\text { Rochester } \\
\text { Epidemiology } \\
\text { Project, Minnesota, } \\
\text { USA }\end{array}$ & $\begin{array}{l}\text { Retrospective, } 14 \\
\text { years follow-up }\end{array}$ & $\begin{array}{l}\text { One hundred and twenty } \\
\text { TED patients, } 17 \mathrm{M} \\
103 \mathrm{~F} \text {, mean age at diagnosis } \\
44.7 \pm 17.4 \text { years. }\end{array}$ & $\begin{array}{l}\text { Progression/ } \\
\text { treatment effect, surgery }\end{array}$ & $\begin{array}{l}\text { Record review and } \\
\text { follow-up } \\
\text { questionnaire,ime period } \\
\text { of smoking assessment } \\
\text { status not stated }\end{array}$ & $\begin{array}{l}\text { Current-smokers vs } \\
\text { non-smokers (not } \\
\text { defined) }\end{array}$ & $\begin{array}{l}\text { Sex, age, thyroid } \\
\text { dysfunction, years since } \\
\text { diagnosis }\end{array}$ & $\begin{array}{l}\text { No significant difference in } \\
\text { outcomes, after adjustment for } \\
\text { confounders: } P=0.73\end{array}$ \\
\hline \multirow[t]{2}{*}{ Bartalena et $a l^{21}$} & $\begin{array}{l}\text { University Hospital } \\
\text { Eye Centre, Italy }\end{array}$ & $\begin{array}{l}\text { Prospective study } \\
\text { nested in RCT of } \\
\text { treatment for GD. } \\
\text { Time period } \\
\text { not stated }\end{array}$ & $\begin{array}{l}\text { Three hundred patients } \\
\text { undergoing radioiodine } \\
\text { treatment for Graves' } \\
\text { hyperthyroidism, mild or } \\
\text { no ophthalmopathy. }\end{array}$ & $\begin{array}{l}\text { Outcome of treatment: } \\
\text { progression/ } \\
\text { improvement of known } \\
\text { cases }\end{array}$ & $\begin{array}{l}\text { Method of data } \\
\text { collection not stated. } \\
\text { Lifelong assessment } \\
\text { of smoking status }\end{array}$ & $\begin{array}{l}\text { Current-smokers } \\
\text { plus ex-smokers } \\
<1 \text { year } v s \text { nonsmokers } \\
\text { plus ex-smokers }>1 \text { year }\end{array}$ & Radioiodine treatment & $\begin{array}{l}\text { Increased progression following } \\
\text { radioiodine treatment: RR } 3.94 \\
\text { (95\% CI 1.41-11.02) } P=0.003 \\
\text { (calc by reviewer) } \\
\text { Decreased improvement with } \\
\text { radioiodine and steroid treatment: } \\
\text { RR 0.23 (95\% CI 0.14-0.40) } P<0.001 \\
\text { (calc by reviewer) }\end{array}$ \\
\hline & & $\begin{array}{l}\text { Retrospective } \\
\text { cohort study of } \\
\text { patients with severe } \\
\text { TED. Time period } \\
\text { not clear }\end{array}$ & $\begin{array}{l}\text { Patients with severe TED } \\
\text { treated with orbital radiation } \\
\text { therapy and high-dose } \\
\text { steroids, } 110 \mathrm{~F} 40 \mathrm{M} \text {, } \\
\text { mean age } 41 \text { (range } 30-63 \text { ) } \\
\text { years }\end{array}$ & $\begin{array}{l}\text { Response } / \text { nonresponse } \\
\text { or progression } \\
\text { (responder }=\text { good/ } \\
\text { excellent } / \text { moderate, } \\
\text { nonresponder }=\text { no } \\
\text { response or progression) }\end{array}$ & $\begin{array}{l}\text { Method of data } \\
\text { collection not stated. } \\
\text { Lifelong assessment of } \\
\text { smoking status }\end{array}$ & $\begin{array}{l}\text { Current-smokers } \\
\text { plus ex-smokers } \\
<1 \text { year } v s \text { non-smokers } \\
\text { plus ex-smokers }>1 \text { year }\end{array}$ & Radioiodine treatment & $\begin{array}{l}\text { Reduced response to therapy: RR } \\
0.73(0.62-0.85) P=0.001 \text { (calc by } \\
\text { reviewer) }\end{array}$ \\
\hline Eckstein $e$ t $a l^{28}$ & $\begin{array}{l}\text { University } \\
\text { Department of } \\
\text { Ophthalmology, } \\
\text { Germany }\end{array}$ & $\begin{array}{l}\text { Prospective, } 1 \text { year } \\
\text { follow-up of patients } \\
\text { with TED }\end{array}$ & $\begin{array}{l}\text { Sixty patients } 41 \\
\text { smokers, } 11 \mathrm{M} 30 \mathrm{~F} \text {, mean } \\
\text { age } 46 \text { (range 18-70) years. } \\
19 \text { non-smokers, } 4 \mathrm{M} 15 \mathrm{~F}, \\
\text { mean age } 47 \text { (range 30-69) } \\
\text { years. }\end{array}$ & $\begin{array}{l}\text { Response to treatment, } \\
\text { clinical activity score } \\
\text { (CAS), motility, proptosis }\end{array}$ & $\begin{array}{l}\text { Level of biomarker, } \\
N \text {-2-hydroxyethylvaline } \\
\text { (HEV) }\end{array}$ & $\begin{array}{l}\text { Current-smokers vs } \\
\text { non-smokers defined } \\
\text { by HEV levels: } \\
\text { current-smoker }=\mathrm{HEV} \\
>2.9 \mu \mathrm{g} / / 1, \text { non- } \\
\text { smoker }=\mathrm{HEV} \\
<2.9 \mu \mathrm{g} / / 1\end{array}$ & $\begin{array}{l}\text { Age, thyroid function, } \\
\text { duration, and severity } \\
\text { of thyroid eye disease }\end{array}$ & $\begin{array}{l}\text { Less improvement in CAS at } \\
12 \text { months } 53 \% \text { of smokers and } \\
74 \% \text { non-smokers, } P<0.05 \\
\text { Less improvement in motility at } \\
12 \text { month } 24 \% \text { smokers and } \\
60 \% \text { non-smokers, } P<0.017 \\
\text { No significant difference in } \\
\text { improvement in proptosis at } \\
12 \text { months, } 9 \% \text { smokers and } \\
5 \% \text { non-smokers, } P=\text { NS }\end{array}$ \\
\hline
\end{tabular}


case-control studies where a quality assessment could be made, at least partially met the criterion for the appropriateness of the controls, although none fully met this criteria. The most common problem was the use of hospital/clinic controls that may have higher rates of smoking than in the true source population for the cases, potentially reducing the size of the observed association between smoking and TED.

Among the cohort studies, only one was considered to at least partially meet all five key criteria assessed. ${ }^{16}$ The other cohort studies only met two or three key quality criteria. The main analysis method was considered adequate in only two studies. ${ }^{16,28}$ The process of allocating subjects to distinct smoking categories was particularly poorly performed in the cohort studies. The strongest approach is to divide subjects into currentsmokers, ex-smokers, and never-smokers, and compare current- and ex-smokers separately with never-smokers in the analysis. Only one study did this, ${ }^{16}$ whereas combining ex-smokers with smokers in a comparison of ever-smokers and never-smokers, or combining ex-smokers with non-smokers in a comparison of current-smokers and current non-smokers was common. Three studies categorised smokers who had stopped within 5 years of the study as current-smokers and those who had stopped more than 5 years previously as non-smokers. ${ }^{23,31,32}$ Three studies categorised patients who had stopped more than 1 year previously as non-smokers. ${ }^{21,27,29}$

Imprecision in exposure assessment causing nondifferential misclassification is a frequent problem in epidemiological studies and tends to bias the observed association of smoking and TED towards the null. The limited nature of the smoking data was a key weakness of the cohort and case-control studies. Lifelong smoking status was assessed in seven studies. ${ }^{16,21,22,25-}$ 27,32 The other studies only assessed current smoking status or status at the onset of disease $e^{5,23,29,31}$ or the method was not stated. ${ }^{24,30}$ A further potential bias in cohort is measurement imprecision where smokers and non-smokers are categorised at baseline only. This will not allow for the effect of changed exposure status, such as quitting smoking, over time. Changes in exposure status are especially likely with prolonged follow-up, and will bias the measure of association towards the null. Only one of the cohort studies re-assessed smoking status during follow-up, ${ }^{16}$ although in most studies where it was described, the follow-up period was short.

The presence of confounding factors presents a problem for all observational studies. Ten studies addressed confounding by sex ${ }^{5,16,23-26,29-32}$ and 11 by age, $5,16,23-26,28-32$ through design or adjustment in analysis. Other confounders addressed were degree of thyroid dysfunction ${ }^{16,28,30}$ and, in studies examining progression of TED in relation to smoking, the duration and severity of eye disease. ${ }^{28,30}$ Two cohort studies adjusted the analysis for radio-iodine and other treatments. ${ }^{16,21}$

\section{Association between smoking and TED}

Overall, eight of the nine case-control studies found a positive association between smoking and the development of TED for current-smokers compared with non-smokers or never-smokers: OR from 1.94 to 20.2, depending on sex of patients, type of ophthalmopathy, and whether controls had thyroid disease or not (Table 2). In all five case-control studies with the methodologically stronger comparison between TED cases and controls with Graves' disease but no ophthalmopathy, there was a positive association between smoking and TED: OR from 1.94 to 10.1. 5,22,24-26 Of these, in the study by Shine et al, ${ }^{22}$ which met the most key quality criteria, the OR for developing TED in current-smokers compared with never-smokers was 3.78 (95\% CI 1.63-9.05). For seven case-control studies in which control subjects did not have thyroid disease, positive associations between TED and smoking with ORs of up to 20.2 were recorded, ${ }^{5,22-24,26,29,31}$ with an OR of 6.5 in the study in which the highest number of quality criteria were at least partially met. ${ }^{23}$ Only one of these seven studies found no association of TED with smoking. ${ }^{32}$

The cohort studies included a retrospective study with 14 years follow-up ${ }^{30}$ and three prospective studies with 1 or 2 years follow-up. ${ }^{16,27,28}$ One of the cohort papers described two studies: an observational study nested in a randomised controlled trial of treatment for Graves' disease and a retrospective cohort study of patients with severe ophthalmopathy; the time period for follow-up was not clear in this study. ${ }^{21}$

Two of the cohort studies examined the occurrence of new cases of TED in patients with Graves' disease. ${ }^{16,27}$ One study at least partially met all five of the key quality criteria and at least 13 of the 14 quality criteria. ${ }^{16}$ This study found an increased risk of total ophthalmopathy (RR 1.32, 95\% CI 1.06-1.64), proptosis (RR 2.64, 95\% CI 1.79-3.90) and diplopia (RR 3.14, 95\% CI 1.65-5.98) in smokers compared with never-smokers. The other study found no association between smoking and eye disease but included ex-smokers in the non-smoker comparison group. ${ }^{27}$

The remaining four cohort studies examined the effects of smoking on progression or on the outcome of treatment in patients with established TED. ${ }^{21,28,30}$ Three studies found a positive association with currentsmokers having a poorer outcome compared with 
current non-smokers (including former smokers). ${ }^{21,28}$ Bartley et $a l,{ }^{30}$ found no evidence of poorer outcome among smokers after following patients for 14 years, although the smoking status of the comparison groups was poorly defined.

\section{Effects of smoking dose}

Nine studies investigated for a dose-response effect of smoking $5,16,22,23,26-28,31,32$ and seven found evidence of a dose response. The strongest analyses were in three studies which assessed the association between a measure of smoking dose (severity and/or duration) and outcome. ${ }^{16,27,28}$ Two cohort studies examined the effect of smoking dose on the development of TED in patients with Graves' disease. ${ }^{16,27}$ The most comprehensive analysis was conducted by Pfeilschifter and Ziegler, ${ }^{16}$ who divided current-smokers by severity of smoking: 1-10 cigarettes/day, 11-20 cigarettes/day, 11-20 cigarettes/day with $<1000000$ total, 11-20 cigarettes/day with $>100000$ total, and $>20$ cigarettes/ day. Compared with never-smokers, in linear trend analysis, there was a significant increase in RR for the development of total symptoms and proptosis $(P<0.05)$ and for diplopia $(P<0.01)$ as smoking dose increased. However, a second methodologically weaker study ${ }^{27}$ found no correlation between development of TED and a smoking index, calculated as the number of daily cigarettes smoked at study entry multiplied by number of years smoking. Eckstein $e t a l^{28}$ followed patients with pre-existing TED and found a significant correlation between $N-2$ hydroxyethylvaline (a marker that reflects the smoking dose over several months previously) and progression as measured by a clinical activity score (a measure of TED progression) and reduced eye motility at $1.5,4.5$, and 7.5 months.

Two studies noted that there were more heavy smokers in groups with severe or moderate/severe TED compared with those with less severe ophthalmopathy. ${ }^{5,22}$ Three studies observed that the OR for development were higher when more severe forms of TED were considered. ${ }^{23,26,32}$ In contrast, one study noted no difference in the percentage of smokers in slight or moderate to severe ophthalmopathy patients. ${ }^{31}$

\section{Evidence for reversibility on smoking cessation}

Reversibility of the association between smoking and TED can be inferred from reduced associations with TED among ex-smokers compared with current-smokers. Among the case-control studies with a comparison between TED cases and Graves' disease controls, Hagg and Asplund ${ }^{5}$ found an OR of 10.0 for the association of TED and smoking for current-smokers vs non-smokers.
This dropped to an OR of 4.5 when current-smokers were combined with ex-smokers and compared with neversmokers. O'Hare and Geoghegan ${ }^{25}$ found a stronger association with current smoking (OR 3.48) at the onset of TED than with ever-smoking (OR 2.29). One cohort study, ${ }^{16}$ the strongest methodologically, examined the effect of stopping smoking on TED and found no increased risk for ophthalmopathy in ex-smokers compared with never-smokers for a variety of indicators: total ophthalmopathy (RR 1.45, 95\% CI 0.90-2.33, $P=0.122)$, proptosis (RR $0.90,95 \%$ CI $0.24-2.33$, $P=0.873$ ), and diplopia (RR 1.88, 95\% CI 0.46-7.73, $P=0.382$ ). This contrasted with the strong association with current smoking.

\section{Discussion}

We found 15 case-control and cohort studies which investigated an association between tobacco smoking and TED. The quality of the studies was variable, with many studies having deficiencies in key quality criteria, and many of the case-control studies having an inappropriate control group to assess the research question adequately. However, most of the studies, particularly those with the strongest designs and methodologies, supported an association between smoking and the development and progression of TED.

Our review had important strengths. In comparison with three previous reviews on this topic, ${ }^{7-9}$ we used a more rigorous search strategy and identified studies not included in these previous reviews. ${ }^{25,26,28,30,32} \mathrm{We}$ included non-English studies. We only included evidence from study designs that are the strongest for investigating causality, and unlike previous reviews, we included a comprehensive assessment of study quality. Because of the small number of high-quality studies, meta-analysis was not considered to be appropriate.

Criteria for the assessment of causality were advanced by $\mathrm{Hill}^{33}$ and subsequently modified by Susser. ${ }^{34}$ The criteria of causality advocated include a strong and consistent association, evidence of dose response and reversibility, a clear temporal relationship, and biological plausibility.

We found evidence of a strong and consistent association between smoking and TED. Eight of nine case-control studies found a positive association with an up to 20-fold increased risk of TED for current-smokers compared with non- or never-smokers. However, in four studies, analysis was restricted only to a comparison of cases with TED and controls without Graves' disease, which makes the results more difficult to interpret. All five of the case-control studies which carried out the more appropriate comparison of smoking among patients with Graves' disease with and without TED 
found evidence of an association, including the study which met the greatest number of the key quality criteria.

Prospective cohort studies are the best placed to demonstrate unequivocally that smoking preceded the development or progression of TED. Of the two prospective studies investigating causes of incident TED, the study which was judged to be of highest quality found a strong positive association between smoking and the incidence of TED in patients with Graves' disease. ${ }^{16}$ The second such study found no association, but was methodologically weaker. There was also an association of smoking with greater progression of TED and/or poorer response to treatment in established TED: three of four studies finding a positive association.

Evidence came from studies which were heterogeneous in setting, design, and analysis. Studies assessed the association of smoking in populations from Europe, America, and the Far East; in case-control and cohort studies; for incident or prevalent TED; and progression or treatment outcome. Controls for the case-control studies included hospital and community controls with or without Graves' disease. TED is not a homogeneous diagnosis and definitions of disease varied between studies, with different classifications, symptoms, and severities of disease.

Most studies which investigated for it showed a doseresponse effect with increased incidence of TED and severity of eye disease symptoms as smoking intensity increased and stronger associations with smoking in patients with severe TED compared with less severe disease. Only three studies examined the effects of quitting smoking. All found evidence of reduced risk for TED among ex-smokers compared with current smokers.

The biological mechanisms by which smoking contributes to the development of TED remain unclear. Several theories have been proposed. For example, smoking may have a direct irritant action on the eye. This would explain the inflammatory changes, but does not explain the increased volume of extraocular muscle and retrobulbar fibroadipose tissues. ${ }^{1}$ Smoking has been linked to other autoimmune diseases including rheumatoid arthritis and Crohn's disease; this suggests there may be a generalised stimulation of the autoimmune process in smokers. ${ }^{2}$ Cytokines are believed to play an important role in the pathogenesis of TED and smoking might influence cytokine secretion and activity by causing hypoxia in the retrobulbar space. ${ }^{1-3}$ Other postulated mechanisms include smoking affecting immunological reactions involved in the pathogenesis of eye disease by altering TSH and making it more immunogenic, or by sensitising the orbital tissue to an as yet unidentified trigger for TED. ${ }^{1}$

In conclusion, we found strong epidemiological evidence for a causal association between tobacco smoking and TED, with RR/OR $>2$ in most studies. The quality of the studies was variable but evidence of association was most consistently present in the higher quality studies. Similar associations have also been found for smoking and the development of age-related macular degeneration ${ }^{35}$ and cataract. ${ }^{36}$ In our judgement on the balance of the evidence available, particularly from the higher quality studies, there is sufficient evidence of causality and hence justification for educational and smoking cessation interventions with patients and the public who are often unaware that smoking has adverse ocular effects. ${ }^{37}$ Patients with Graves' disease should be encouraged to stop smoking in order to both help prevent development of TED and to reduce the general physical harm that smoking causes. Patients with existing TED should also be advised to stop smoking, as smoking cessation is likely to slow or stop progression of eye disease and improve the outcome of treatment. Many diabetes, cardiac, and respiratory clinics have incorporated smoking cessation support into their routine services. UK ophthalmology or optometry services and endocrine clinics should likewise routinely assess smoking status and offer smoking cessation support for patients with or at risk of eye diseases related to smoking.

\section{Acknowledgements}

This study was supported by research funds at Bolton Eye Unit, Bolton Hospitals NHS Trust. We thank Mr M Potts for his helpful comments on the draft manuscript.

\section{References}

1 Bartalena L, Pinchera A, Marcocci C. Management of Graves' ophthalmopathy: reality and perspectives. Endocr Rev 2000; 21: 168-199.

2 Prabhakar BS, Bahn RS, Smith TJ. Current perspective on the pathogenesis of Graves' disease and ophthalmopathy. Endocr Rev 2003; 24: 802-835.

3 Bartalena L, Marcocci C, Pinchera A. Graves 'ophthalmopathy: a preventable disease? Eur J Endocrinol 2002; 146: 457-461.

4 Cawood T, Moriarty P, O'Shea D. Recent developments in thyroid eye disease. BMJ 2004; 329: 385-390.

5 Hagg E, Asplund K. Is endocrine ophthalmopathy related to smoking. BMJ 1987; 295: 634-635.

6 Krassas GE, Wiersinga W. Smoking and autoimmune thyroid disease: the plot thickens. Eur J Endocrinol 2006; 154: 777-780.

7 Solberg Y, Rosner M, Belkin M. The association between cigarette smoking and ocular diseases. Surv Ophthalmol 1998; 42: 535-547.

8 Hegedus L, Brix TH, Vestergaard P. Relationship between cigarette smoking and Graves' ophthalmopathy. J Endocrinol Invest 2004; 27: 265-271.

9 Vestergaard P. Smoking and thyroid disorders - a metaanalysis. Eur J Endocrinol 2002; 146: 153-161. 
10 Silman AJ, MacFarlane GJ. Studying associations between risk factors and disease. In: Silman AJ, MacFarlane GJ (eds). Epidemiological Studies: A Practical Guide. Cambridge University Press: Cambridge, 2002, pp 31-41.

11 Deeks JJ, Dinnes J, D’Amico R, Sowden AJ, Sakarovitch C, Song $\mathrm{F}$ et al. Evaluating non-randomised intervention studies. Health Technology Assessment (Winchester, England) 2003; 7: iii-x, 1-173.

12 Critical Appraisal Skills Programme. Twelve questions to help you make sense of a cohort study. Update Software: Oxford, 1999.

13 Wells G, Shea B, O'Connell D, Peterson J, Welch V, Losos M et al. The Newcastle-Ottawa Scale (NOS) for assessing the quality of nonrandomised studies in meta-analysis (2006). http://www.ohrc.ca/programs/clinical_epidemiology/ oxford.htm.

14 Downs SH, Black N. The feasibility of creating a checklist for the assessment of the methodological quality both of randomised and non-randomised studies of health care interventions. J Epidemiol Commun Health 1998; 52: 377-384.

15 Friedenreich CM, Brant RF, Riboli E. Influence of methodologic factors in a pooled analysis of 13 case-control studies of colorectal cancer and dietary fiber. Epidemiology 1994; 5: 66-79.

16 Pfeilschifter J, Ziegler R. Smoking and endocrine ophthalmopathy: impact of smoking severity and current vs lifetime cigarette consumption. Clin Endocrinol 1996; 45: 477-481.

17 Szucs-Farkas Z, Toth J, Kollar J, Galuska L, Burman KD, Boda $\mathrm{J}$ et al. Volume changes in intra- and extraorbital compartments in patients with Graves' ophthalmopathy: effect of smoking. Thyroid 2005; 15: 146-151.

18 Marquez SD, Lum BL, McDougall IR, Katkuri S, Levin PS, MacManus $\mathrm{M}$ et al. Long-term results of irradiation for patients with progressive Graves' ophthalmopathy. Int $J$ Radiat Oncol Biol Phys 2001; 51: 766-774.

19 Tagami T, Tanaka K, Sugawa H, Nakamura H, Miyoshi Y, Mori T et al. High-dose intravenous steroid pulse therapy in thyroid-associated ophthalmopathy. Endocr J 1996; 43: 689-699.

20 Jensen BE, Bonnema SJ, Hegedus L. Glucocorticoids do not influence the effect of radioiodine therapy in Graves' disease. Eur J Endocrinol 2005; 153: 15-21.

21 Bartalena L, Marcocci C, Tanda ML, Manetti L, Dell'Unto E, Bartolomei MP et al. Cigarette smoking and treatment outcomes in Graves ophthalmopathy. Ann Int Med 1998; 129: 632-635.
22 Shine B, Fells P, Edwards OM, Weetman AP. Association between Graves' ophthalmopathy and smoking. Lancet 1990; 335: 1261-1263.

23 Prummel MF, Wiersinga WM. Smoking and risk of Graves' disease. JAMA 1993; 269: 479-482.

24 Winsa B, Mandahl A, Karlsson FA. Graves' disease, endocrine ophthalmopathy and smoking. Acta Endocrinol 1993; 128: 156-160.

25 O'Hare JA, Geoghegan M. Cigarettes smoking as a promoter of Graves' disease. Eur J Int Med 1993; 4: 289-292.

26 Bartalena L, Martino E, Marcocci C, Bogazzi F, Panicucci M, Velluzzi F et al. More on smoking habits and Graves' ophthalmopathy. J Endocrinol Invest 1989; 12: 733-737.

27 Tallstedt L, Lundell G, Taube A. Graves' ophthalmopathy and tobacco smoking. Acta Endocrinol 1993; 129: 147-150.

28 Eckstein A, Quadbeck B, Mueller G, Rettenmeier AW, Hoermann R, Mann K et al. Impact of smoking on the response to treatment of thyroid associated ophthalmopathy. Br J Ophthalmol 2003; 87: 773-776.

29 Nunery WR, Martin RT, Heinz GW, Gavin TJ. The association of cigarette smoking with clinical subtypes of ophthalmic Graves' disease. Ophthalmic Plast Reconstru Surg 1993; 9: 77-82.

30 Bartley GB, Fatourechi V, Kadrmas EF, Jacobsen SJ, Ilstrup DM, Garrity JA et al. The treatment of Graves' ophthalmopathy in an incidence cohort. Am J Ophthalmol 1996; 121: 200-206.

31 Chen YL, Chang TC, Chen CJ. Influence of smoking on Graves' disease with or without ophthalmopathy and nontoxic nodular goiter in Taiwan. J Formos Med Assoc 1994; 93: 40-44.

32 Shiraishi S, Chuman H, Nao-i N, Sawada A, Ohzuno I. Influence of smoking on hyperthyroid ophthalmopathy. Jpn J Clin Ophthalmol 1997; 51: 899-901.

33 Hill AB. The environment and disease: association or causation? Proc $R$ Soc Med 1965; 58: 295-300.

34 Susser M. What is a cause and how do we know one? A grammar for pragmatic epidemiology. Am J Epidemiol 1991; 133: 635-648

35 Thornton J, Edwards R, Mitchell P, Harrison RA, Buchan I, Kelly SP. Smoking and age-related macular degeneration: a review of association. Eye 2005; 19: 935-944.

36 Kelly SP, Thornton J, Edwards R, Sahu A, Harrison R. Smoking and cataract: review of causal association. J Cataract Refract Surg 2005; 31: 2395-2404.

37 Bidwell G, Sahu A, Edwards R, Harrison RA, Thornton J, Kelly SP. Perceptions of blindness related to smoking: a hospital-based cross-sectional study. Eye 2005; 19: 945-948. 